\title{
Partition-and-Shift LDPC Codes
}

\author{
Jin $\mathrm{Lu}^{1}$, Member, IEEE, and José M. F. Moura ${ }^{2}$, Fellow, IEEE \\ ${ }^{1}$ Sun Microsystems, Lafayette, CO 80026 USA \\ ${ }^{2}$ Department of Electrical and Computer Engineering, Carnegie Mellon University, Pittsburgh, PA 15213-3890 USA
}

\begin{abstract}
This paper describes a new type of regular structured low-density parity-check (LDPC) code: the partition-and-shift LDPC (PS-LDPC) code. PS-LDPC codes can be easily designed to have large girth. The code construction is simple to explain: we divide the bit and check nodes in the Tanner graph into subsets and connect nodes in these subsets according to a set of parameters called shifts. We derive a general theorem on the shifts to prevent cycles that are harmful to LDPC decoding. This theorem provides a methodology to design PS-LDPC codes with arbitrary column weight $j$ and large girth $g$. Simulation results over EPR4 channels demonstrate the good bit-error rate performance of PS-LDPC codes.
\end{abstract}

Index Terms-Error floor, girth, low-density parity-check (LDPC).

\section{INTRODUCTION}

H IGH-RATE low-density parity-check (LDPC) codes are the focus of intense research in magnetic recording because, when decoded by the iterative sum-product algorithm, they show performance close to the Shannon Capacity [1]. LDPC codes can be described by a bipartite graph called a Tanner graph [2]. The length of the shortest cycle in a Tanner graph is referred to as its girth $g$. Since large girth improves the bit-error performance of the codes, leads to more efficient decoding, and guarantees large minimum distance [2], LDPC codes with large girth are particularly desired.

Structured LDPC codes reduce the encoder and decoder complexities. We only discuss here a few typical techniques to design structured LDPC codes. Reference [3] designs LDPC codes based on finite geometries. Codes constructed in this way have girth 6. Another well-known method to construct structured regular LDPC codes is based on balanced incomplete block designs (BIBD) [4], [5]. BIBD-based codes are well structured, free of 4-cycles, i.e., their girth is $g=6$, and achieve a very high code rate. For a more expansive review of work on structured LDPC codes, please refer to the overviews in [6] and [7].

In this paper, we describe a class of structured regular LDPC codes with large girth and high code rates, called partition-and-shift LDPC (PS-LDPC) codes, and explore their error-correcting performance in magnetic recording channels.

\section{Code Construction}

We briefly introduce PS-LDPC codes. For their detailed construction please refer to [8] and [9]. Let $V_{c}$ be the set of all check nodes and $V_{b}$ the set of all bit nodes in a Tanner graph. Divide $V_{c}$ into $N_{c}$ disjoint subsets of equal size provided that the code block length $n=N_{c} \cdot p$, where $p$ is a natural number (not necessarily a prime number). We index the check nodes in each subset from 0 to $p-1$. Similarly, partition $V_{b}$ into $N_{b}$ disjoint subsets of equal size and index the bit nodes in each subset from 0 to $p-1$.

PS-LDPC codes, as defined in [8] and [9], are LDPC codes that satisfy the following assumptions.

Digital Object Identifier 10.1109/TMAG.2005.854446

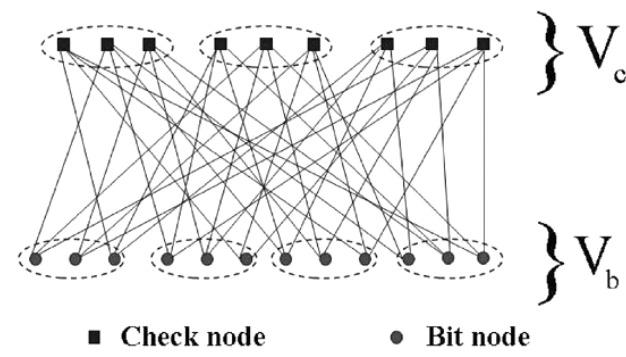

Fig. 1. Tanner graph for PS-LDPC code.

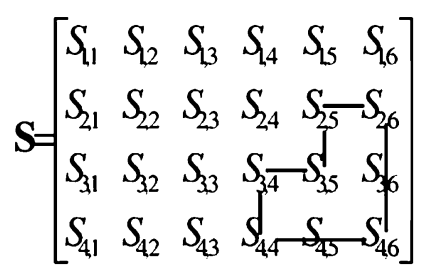

(a)

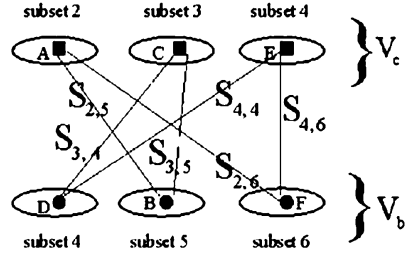

(b)
Fig. 2. (a) Closed path in shift matrix $\mathbf{S}$. (b) Six-cycle in Tanner graph with six associated shifts $S_{2,5}, S_{3,5}, S_{3,4}, S_{4,4}, S_{4,6}, S_{2,6}$.

1) Check nodes: Each check node is connected to $k$ bit nodes in $k$ different bit node subsets. The check nodes in the same check node subset are connected to distinct bit nodes in the same $k$ bit node subsets.

2) Bit nodes: Each bit node is connected to $j$ check nodes in $j$ different check node subsets. The bit nodes in the same bit node subset are connected to distinct check nodes in the same $j$ check node subsets.

3) Shifts: Every check node, indexed by $X$ in the $\alpha$ th check node subset, is connected to the bit node indexed by $W$ in the $\beta$ th bit node subset, where $W=X \stackrel{p}{\oplus} S_{\alpha, \beta}$ and $0 \leq S_{\alpha, \beta} \leq p-1$.

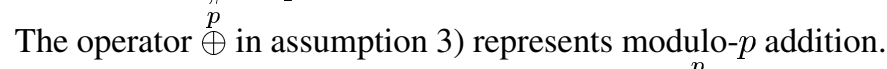
Similarly, we represent modulo- $p$ subtraction by $\stackrel{p}{\ominus}$. The parameters $S_{\alpha, \beta}, \alpha=1, \ldots, N_{c}, \beta=1, \ldots, N_{b}$ in assumption 3) are called shifts. We collect all the shifts $S_{\alpha, \beta}$ in an $N_{c} \times N_{b}$ matrix called the shift matrix $\mathbf{S}=\left[S_{\alpha, \beta}\right]$. For example, Fig. 2(a) shows a shift matrix. Fig. 1 shows the Tanner graph for a PS-LDPC code where all the check nodes are divided into three check node subsets and all the bit nodes are partitioned into four-bit node 
subsets. In each subset (check node subset or bit node subset), there are three nodes.

Code Rate: We can design PS-LDPC codes with any desired values for the column weight $j$ and the row weight $k$. For a regular code, ignoring the effect of a few linearly dependent parity-check equations, the code rate is well approximated by $\rho=1-(j / k)$, which means that we can generate PS-LDPC codes with any practical desired code rate by simply choosing appropriate values for $j$ and $k$. For example, if a $\rho^{*}=8 / 9$ and column-weight-3 PS-LDPC code is desired, set $j=3$ and $k=27$.

\section{CYCLES AND SHIFTS}

By construction, PS-LDPC codes are characterized by shifts $S_{\alpha, \beta}, \alpha=1, \ldots, N_{c}, \beta=1, \ldots, N_{b}$. Since our goal is to eliminate those short cycles in Tanner graphs, we first relate $2 t$ cycles $(t \in \mathbb{N})$ in Tanner graphs to shifts $S_{\alpha, \beta}$.

Before presenting this relationship, we first introduce notation. A closed path in $\mathbf{S}$ is defined to be a sequence of connected alternating vertical and horizontal lines such that the last line in the sequence terminates at the beginning of the first line and vertices of the closed path are entries of $\mathbf{S}$. The length of a closed path $\mathbf{L}$ is the number of lines $\mathbf{L}$ it contains. As an illustration, Fig. 2(a) depicts a closed path (solid line) in a shift matrix $\mathbf{S}$. Its six vertices are $S_{2,5}, S_{3,5}, S_{3,4}, S_{4,4}, S_{4,6}$, and $S_{2,6}$. Since this closed path contains six lines, its length is six.

Next, we show the correspondence between cycles in Tanner graphs and shifts in the following theorem.

Theorem 1 (2t-CYCLE): The Tanner graph for a PS-LDPC code contains at least one $2 t$ cycle if and only if there exists a closed path of length $2 t$ in the shift matrix $\mathbf{S}$ such that its $2 t$ vertices $S_{\alpha_{1}, \beta_{1}} \ldots S_{\alpha_{2 t}, \beta_{2 t}}$ satisfy the shift condition $\oplus_{i=1}^{2 t}(-1)^{i+1} S_{\alpha_{i}, \beta_{i}}=0$

$\left(\oplus_{i=1}^{2 t}(-1)^{i+1} S_{\alpha_{i}, \beta_{i}}=S_{\alpha_{1}, \beta_{1}} \stackrel{p}{\ominus} S_{\alpha_{2}, \beta_{2}} \stackrel{p}{\oplus} \cdots\right.$

$\left.\stackrel{p}{\oplus} S_{\alpha_{2 d-1}, \beta_{2 d-1}} \stackrel{p}{\ominus} S_{\alpha_{2 d}, \beta_{2 d}} \stackrel{p}{\oplus} \cdots \stackrel{p}{\oplus} S_{\alpha_{2 t-1}, \beta_{2 t-1}} \stackrel{p}{\ominus} S_{\alpha_{2 t}, \beta_{2 t}}\right)$. [8].

Proof: We omit the proof here. For details, please refer to

We illustrate Theorem 1 with an example. As shown in Fig. 2(a), the six shifts $S_{2,5}, S_{3,5}, S_{3,4}, S_{4,4}, S_{4,6}$, and $S_{2,6}$ are corners of a closed path of length six (solid line) in S. By Theorem 1, if the shift condition is violated, i.e., $S_{2,5} \stackrel{p}{\ominus} S_{3,5} \stackrel{p}{\oplus} S_{3,4} \stackrel{p}{\ominus} S_{4,4} \stackrel{p}{\oplus} S_{4,6} \stackrel{p}{\ominus} S_{2,6} \neq 0$, then the six-cycle shown in Fig. 2(b) does not exist in the Tanner graph. Similarly, by choosing appropriate shifts $S_{\alpha, \beta}$ for $\alpha=1, \ldots, N_{c}$ and $\beta=1, \ldots, N_{b}$ that violate the shift condition in Theorem 1, we can eliminate cycles with length $2 t, t=2,3,4, \ldots$.

When $N_{c}=j$ and $N_{b}=k$, where $j$ and $k$ are column and row weights of the parity-check matrix, respectively, by choosing appropriate shifts $S_{\alpha, \beta}$ we can design PS-LDPC codes with girth up to 12 . For larger $N_{c}$ and $N_{b}$, we can generate PS-LDPC codes with higher girth [8].

$$
\mathbf{S}=\left[\begin{array}{cccccc}
- & - & - & - & - & - \\
- & - & - & - & - & - \\
- & - & - & - & - & -
\end{array}\right]
$$

Fig. 3. Constructing $\mathbf{S}$ matrix: Step 1.

$$
\mathbf{S}=\left[\begin{array}{cccccc}
80-125 & 105 & 104 & 143 & 25 \\
109-? & - & - & - & - \\
46 & - & - & - & - & -
\end{array}\right]
$$

Fig. 4. Constructing $\mathbf{S}$ matrix: Step 2.

$$
\mathbf{S}=\left[\begin{array}{cccccc}
80 & 125 & 105 & 104 & 143 & 25 \\
109 & 85 & 81 & 93 & 80 & 4 \\
46 & 55 & 66 & 119 & 141 & 135
\end{array}\right]
$$

Fig. 5. Constructing $\mathbf{S}$ matrix: Step 3.

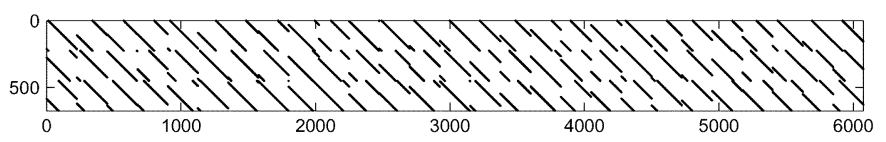

Fig. 6. $\quad \mathbf{H}$ for a $(6075,3,27)$ PS-LDPC code with rate $8 / 9$ and girth 8 .

\section{DESIGN OF PS-LDPC CODES With LARGE GIRTH}

We present next an example to illustrate how to find a shift matrix $\mathbf{S}=\left[S_{\alpha, \beta}\right]$ that avoids cycles of length up to $g-2$.

We want to build a $3 \times 6$ shift matrix $\mathbf{S}$ whose associate PS-LDPC code (its Tanner graph) has girth 10 . The size $p$ of each node subset is set to be 150 .

Initially, all the 18 shifts in the shift matrix $\mathbf{S}$ are undetermined, as shown in Fig. 3.

In the next step, we randomly choose values in between 0 and 149 for the entries in the first row and first column of $\mathbf{S}$. Next, we determine the value of $S_{2,2}$. Since $S_{2,2}$ forms a length-four closed path (solid line in Fig. 4) with the already existing shifts $S_{1,1}, S_{1,2}$, and $S_{2,1}$, we need to check whether the four shifts $S_{2,2}, S_{1,1}, S_{1,2}$, and $S_{2,1}$ violate the shift condition in Theorem 1. For instance, we choose $S_{2,2}=4$, then $S_{1,1} \stackrel{p}{\ominus} S_{2,1} \stackrel{p}{\oplus} S_{2,2} \stackrel{p}{\ominus}$ $S_{1,2}=\bmod (80-109+4-125150)=0$. Hence, the shift condition is met. We have to choose another value of $S_{2,2}$. Say this time we choose $S_{2,2}=85$, then $S_{1,1} \stackrel{p}{\ominus} S_{2,1} \stackrel{p}{\oplus} S_{2,2} \stackrel{p}{\ominus}$ $S_{1,2}=\bmod (80-109+85-125150)=81$. Hence, the shift condition is violated. We fix the value of $S_{2,2}$ to be 85 . This process is shown in Fig. 4.

This process is repeated until we finally generate a shift matrix $\mathbf{S}$ that is free of cycles shorter than ten, as shown in Fig. 5.

After finding a shift matrix $\mathbf{S}$, we can construct a PS-LDPC code from the resulting $\mathbf{S}$ matrix.

Similarly, Fig. 6 shows the $675 \times 6075$ parity-check matrix $\mathbf{H}$ for a $(6075,3,27)$ PS-LDPC code with rate $r=8 / 9$ and girth 8 . The matrix $\mathbf{H}$ is developed from a $3 \times 27 \mathbf{S}$ matrix generated by the method described above.

As stated before, PS-LDPC codes can be designed with large girth and any desired code rate. However, by forcing PS-LDPC codes to have large girth, we lose some flexibility in choosing the block size. A PS-LDPC code with column weight $j$, row 


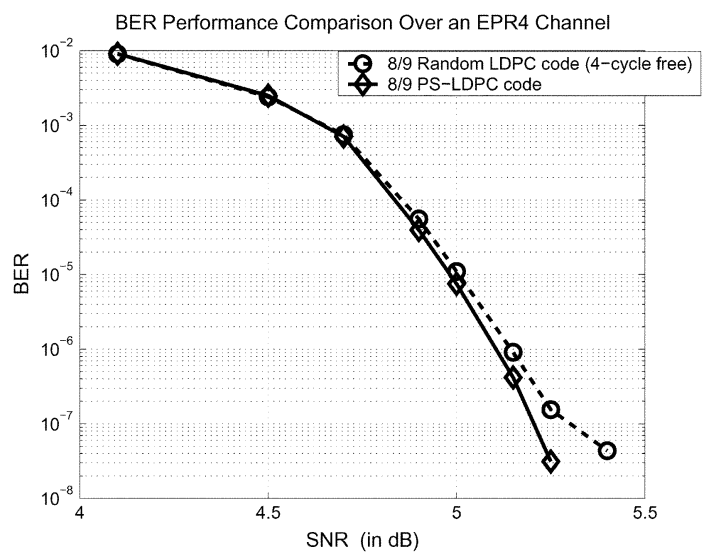

Fig. 7. BER performance: PS-LDPC code, column weight 3 , girth 8 , and rate $8 / 9$ versus same size random LDPC code free of four cycles.

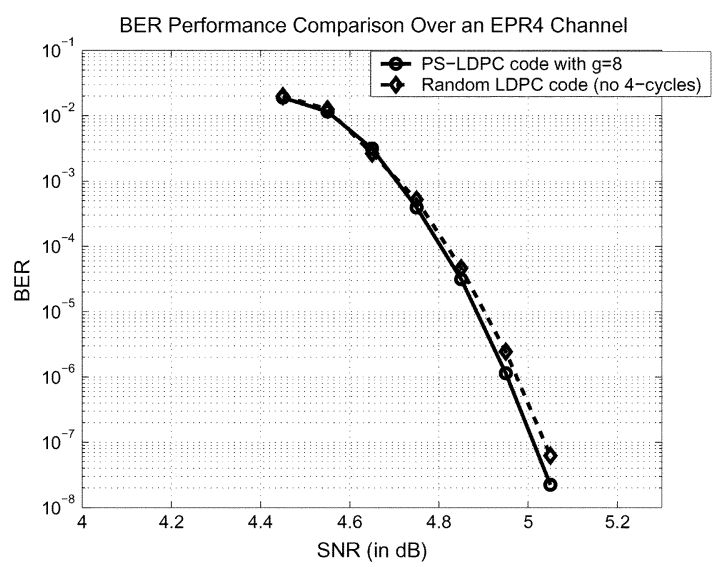

Fig. 8. BER performance: PS-LDPC code, column weight 4 , girth 8 , and rate $8 / 9$ versus same size random LDPC code free of four cycles.

weight $k$, girth $g$, and block size $n$ should follow the bound proposed in [10]: $n>[(j-1)(k-1)]^{\lfloor(g-2) /(4)\rfloor}$. The exact value of code block size $n$ can be found by computer search.

\section{Simulation Results}

We study by simulation the bit-error rate (BER) of large-girth PS-LDPC codes in EPR4 channels. For the purpose of comparison, we also show the BER of random LDPC codes free of four cycles [1] that have the same size and same code rate as the PS-LDPC codes we constructed. The signal-to-noise ratio (SNR) definition we use is $\mathrm{SNR}=10 \log _{10}\left[E_{b} / N_{0}\right]$, where $E_{b}$ denotes the user bit energy and $N_{0} / 2$ is the two-sided power spectral density.

Fig. 7 shows the BER of a PS-LDPC code with girth 8 and the BER of a fiour-cycle free-random LDPC code in an EPR4 channel. Both codes have column weight $j=3$, block length 6075 , and code rate 8/9. In the high SNR, the PS-LDPC code outperforms the random LDPC code when BER is less than $10^{-7}$. The plots show that in this region the performance of the random code has shown the omen of an error floor, while the PS-LDPC code has not yet reached the error floor at this BER. We also notice that at low to moderate SNR, PS-LDPC codes have a performance comparable to that of a random LDPC codes free of four cycles.

We have studied in Fig. 7 PS-LDPC codes with column weight $j=3$. Next, we study PS-LDPC codes with column weight $j=4$. The plot in Fig. 8 compares the BER performance for a column weight $j=4$ PS-LDPC code with girth 8 (solid line) with a random LDPC code (no four cycles) with column weight 4 (dashed line). Both codes have the same block length 32760 and the same code rate 8/9. The slope of the BER curve for the PS-LDPC code decreases faster with SNR than that of random LDPC code. At low to moderate SNR, both codes have similar BER performance.

\section{CONCLUSION}

We present PS-LDPC codes in this paper. They are particularly suitable for future high-density data storage systems due to the following reasons demonstrated in the paper.

1) They can be systematically constructed with high code rates and large girth.

2) Their parity-check matrix is based on a much smaller matrix - the shift matrix $\mathbf{S}$, which reduces the memory requirements in hardware implementation.

3) Their BER performance outperforms random codes at high SNR. We observe that they can alleviate the error floor.

\section{ACKNOWLEDGMENT}

This work was supported by the Data Storage Systems Center (DSSC), Carnegie Mellon University.

\section{REFERENCES}

[1] D. J. C. Mackay, "Good error-correcting codes based on very sparse matrices," IEEE Trans. Inf. Theory, vol. 45, no. 2, pp. 399-431, Mar. 1999.

[2] R. M. Tanner, "A recursive approach to low complexity codes," IEEE Trans. Inf. Theory, vol. IT-27, no. 5, pp. 533-547, Sep. 1981.

[3] Y. Kou, S. Lin, and M. P. C. Fossorier, "Low-density parity-check codes based on finite geometries: A rediscovery and new results," IEEE Trans. Inf. Theory, vol. 47, no. 7, pp. 2711-2736, Nov. 2001.

[4] B. Vasic, E. M. Kurtas, and A. V. Kuznetsov, "Kirkman systems and their application in perpendicular magnetic recording," IEEE Trans. Magn., vol. 38, no. 4, pp. 1705-1710, Jul. 2002.

[5] B. Vasic, E. M. Kurtas, and A. V. Kuznetsov, "LDPC codes based on mutually orthogonal Latin rectangles and their application in perpendicular magnetic recording," IEEE Trans. Magn., vol. 38, no. 5, pp. 2346-2348, Sept. 2002.

[6] B. Vasic and O. Milenkovic, "Combinatorial constructions of low-density parity-check codes for iterative decoding," IEEE Trans. Inf. Theory, vol. 50, no. 6, Jun. 2004.

[7] J. M. F. Moura, J. Lu, and H. Zhang, "Structured LDPC codes with large girth," IEEE Signal Processing Mag., vol. 21, no. 1, Jan. 2004.

[8] J. Lu, J. M. F. Moura, and U. Niesen, "PS-LDPC Codes: Regular structure and arbitrary girth," submitted for publication.

[9] J. Lu, J. M. F. Moura, and U. Niesen, "Grouping-and-shifting designs for structured LDPC codes with large girth," in Proc. ISIT 2004, Chicago, IL, Jun. 27-July 22004

[10] R. G. Gallager, Low-Density Parity Check Codes. Cambridge, MA: MIT Press, 1963.

Manuscript received February 8, 2005. 